\title{
GPs' views on prioritisation of child and adolescent mental health problems
}

\author{
Stephen M. Jones and Bindumadhava R. Bhadrinath
}

\begin{abstract}
The demand for child and adolescent psychiatric services outstrips the supply of resources, leaving a large unmet demand. One way of managing this is to priortise referrals. A sample of Norfolk general practitioners were interviewed face to face. They were asked to prioritise child and adolescent mental health problems that might present to them in the surgery. A high response rate was obtained. Anxiety provoking problems were considered to be of the highest priority. Service provision and prioritisation should take cognisance of the wishes of referrers themselves. Mental health care training priorities in general practice include substance misuse and psychiatric emergencles.
\end{abstract}

Mental health disorders in childhood and adolescence are believed to affect between 7 and $20 \%$ of individuals, with development or functioning, or both, significantly impaired in 5-10\% (Vanstraelen \& Cottrell, 1994). While severe disorders such as anorexia nervosa, autism and early psychosis are rare, substance misuse is common.

Demands are increasing both from within the health service and allied agencies. Ultimately, either additional resources will be needed to meet the rising expectations, or the work of our service will need to be prioritised, or both.

We work in a community-based child mental health service. Assuming a conservative estimate of $14 \%$ prevalence of mental health problems within the area served, the expected number of children with such problems is approximately 20250 (children and adolescents aged 19 years and under).

The Bethel Child and Family Centre has approximately 2000 active cases leaving a large unmet demand, requiring assigning of priorities. We felt that setting such priorities should be informed by the referrers among others, one such group would be general practitioners (GPs).

The aim of this study was to determine how GPs would prioritise child and adolescent mental health problems. GPs' perceptions of priorities are likely to influence their referring behaviour (McColl et al, 1994; Wolpert \& Fredman, 1996).

\section{The study}

A questionnaire was developed by piloting it with two GPs in different settings and one consultant child and adolescent psychiatrist.

The first author (S.M.J.) held face to face interviews (20 minutes each) with GPs in their surgeries, over a geographical area (Norfolk). A multi-stage cluster sampling was used to select GPs to be interviewed. We used the sectors of the local adult psychiatric services to select a random sample of GP practices from each sector. From each such practice a random sample of GPs ( $n=54)$ was selected.

Demographic details were collected about the GP and their practice, as well as information regarding experience of psychiatry. The GPs were then given a list of child and adolescent mental health problems that might present to them in the surgery. They were asked to prioritise each of these in turn on a scale of $1-6$. They were then asked to assume that: (a) the presenting problem was not treatable; and (b) the presenting problem was known to have a poor treatment response. They were asked in each case to say whether the level of assigned priority of the problem would increase, decrease or remain the same. They had the option to state whether they considered that the problem was not a psychiatric one.

\section{Findings}

Of the 54 GPs sampled, 47 took part, a response rate of $87 \%$. Those GPs taking part expressed a desire to get feedback of our findings. The sample comprised $35(74.5 \%)$ men and $12(25.5 \%)$ women. The primary medical qualification was a United Kingdom degree for the majority (98\%). Thirty-eight $(81 \%)$ of the sample had a postgraduate medical qualification.

The practices sampled appear to be evenly distributed between rural, urban and mixed areas. Twenty-three $(49 \%)$ of the practices were dispensing and $22(47 \%)$ were fundholding. Eighteen (38\%) of the practices were actively involved in teaching GP registrars. 
Forty-three (91\%) GPs had experience of adult psychiatry as an undergraduate while $20(43 \%)$ GPs had experience of child and adolescent psychiatry, a ratio of approximately $2: 1$.

Seventeen (36\%) GPs said they have a special interest in psychiatry. However, only two (4\%) held special surgeries for psychological problems. As expected, the majority of GPs give patients with psychological problems a longer consultation, usually at the end of a surgery.

In order to show the presenting problems in rank order of priority (Table 1), we weighted the priority codes on the original scale $(1-6)$ in steps of $0.25(1.5-0.25)$. The weighted priority codes were multiplied by the number of GPs for each code to arrive at a total weighted score (range 11.75-70.5).

With regard to how treatability (not treatable and poor treatment response) affects GPs' prioritisation, the following results were obtained. None of the GPs said their prioritisation of the presenting problem would increase if they knew it was not treatable; $13-57 \%$ of the sample said their prioritisation of the presenting problem would decrease. However, the range was 13$19 \%$ for presenting problems involving risk (i.e. act of deliberate self-harm, child abuse and child or adolescent 'unsafe'). GPs felt that such presentations should be shared quickly with a specialist, to share the anxiety and exclude a psychiatric illness. There were only two presenting problems, school non-attendance or breakdown and behavioural disorder, where the majority of GPs would decrease their prioritisation on the basis of the problem being untreatable (57 and $55 \%$, respectively).

Table 1. Presenting problems in rank order of priority

\begin{tabular}{ll}
\hline $\begin{array}{l}\text { Presenting problem } \\
\text { Act of deliberate self-harm by the child }\end{array}$ & $\begin{array}{l}\text { Weighted } \\
\text { score }\end{array}$ \\
$\begin{array}{l}\text { or adolescent } \\
\text { Child abuse (sexual, physical, } \\
\text { emotional or any combination) }\end{array}$ & 66.25 \\
$\begin{array}{l}\text { Child or adolescent is considered to } \\
\text { be currently 'unsafe' }\end{array}$ & 66.25 \\
$\begin{array}{l}\text { Substance misuse (drugs and/or alcohol) } \\
\text { Eating disorders }\end{array}$ & 62.75 \\
Bereavement, separation or other & 48.75 \\
$\quad$ distressing events & 44.25 \\
Emotional disorders & 44.25 \\
Behavioural disorder & 43.25 \\
Offending behaviour & 43 \\
School non-attendance or breakdown & 40.5 \\
Impairment of intellectual functioning & 37.5 \\
Family breakdown and divorce & 36.25 \\
\hline
\end{tabular}

1. At risk of abuse or suicide, or at risk because they may be psychotic.
If there was a known poor treatment response, $0-6 \%$ of GPs said that their prioritisation would increase, this being most noticeable for deliberate self-harm (6\%) and emotional disorders (6\%) and least for behavioural disorder (0\%). However, 4-40\% of GPs said their prioritisation would decrease, this being most noticeable for school non-attendance or breakdown (40\%), behavioural disorder (36\%) and family breakdown and divorce $(36 \%)$ and least noticeable for child abuse (4\%) and deliberate self-harm (6\%).

The GPs were able to code for not a psychiatric problem' when prioritising according to treatability. Adding together the percentage of GPs who entered this code in both scenarios for treatability (no treatment response and poor treatment response), offending behaviour was the presenting problem most likely to be considered 'not a psychiatric problem', followed by school non-attendance or breakdown and family breakdown and divorce. On the other hand, child abuse was always considered to be a psychiatric problem. The other presenting problems fell somewhere in between.

When asked about changes that the GPs would like to see, the following were the most frequent responses (in rank order), being mentioned by at least five different GPs:

(a) the waiting list and therefore waiting time could be reduced:

(b) GPs would like more information about the multi-disciplinary teams;

(c) more 'crisis intervention' should be provided;

(d) feedback to GPs could be improved, for example, less complicated and more focused letters and use of the telephone;

(e) improved service for the 16-18-year-old age group;

(f) more liaison and consultation with GPs, e.g. in their surgeries.

\section{Comment}

This was a Norfolk study investigating the prioritisation of child and adolescent mental health problems by GPs referring to an outpatient child and adolescent psychiatric clinic. On the whole the GPs spoke highly of the existing service, once families are seen. The main misgiving was the time taken for nonurgent referrals to be seen. GPs would want a more rapid response once a family is referred. They also highlighted the particular issue of an improved service for 16-18 year olds (e.g. a local in-patient adolescent unit and access to the help of community psychiatric nurses). GPs expressed a desire to be more actively involved in the care of children and adolescents with mental health problems (e.g. 
through liaison and consultation about complex cases in the GP surgery). They value outreach clinics, where these are provided. Furthermore, many GPs expressed a desire to know the results of our study. We see feedback to all participants as an essential task (Whitfield, 1997).

The order of ranking of presenting problems by GPs is interesting bearing in mind recent articles in the medical literature (e.g. Goodman, 1997). The top three presenting problems were all scenarios where the GP wanted to share the anxiety and the responsibility with a specialist. In practice in many of these situations social services, with their statutory obligations, are the likely lead agency.

Substance misuse (drugs and/or alcohol) was ranked as the third most important priority. There is a scarcity of service provision nationally for substance misusing young people.

School non-attendance or breakdown was given a low priority, particularly if there is no evidence for a treatable disorder.

Impairment of intellectual functioning was given a low priority. Indeed some GPs said that pervasive developmental disorders are not a psychiatric problem. One possible explanation for this is that GPs refer patients with pervasive developmental disorders to paediatricians. Family breakdown and divorce was given the lowest priority of all.

The issue of treatability did not affect prioritisation for problems which are anxiety provoking and which the GPs want to share (e.g. deliberate self-harm). There are only two presenting problems where the majority of GPs would decrease their prioritisation on the basis of untreatability: school non-attendance or breakdown and behavioural disorder.
A similar study of child and adolescent psychiatrists and purchasers would be of considerable value in assisting purchasers to set priorities.

\section{Acknowledgements}

We thank all the general practitioners who gave of their time to take part in this study. Also, Dr Chris Palmer, Director, Centre for Applied Medical Statistics, University of Cambridge and Dr Christopher Hand, Health Policy and Practice Unit. University of East Anglia for their contributions to the planning of the study.

\section{References}

GOODMAN, R. (1997) Who needs child psychiatrists? Child Psychology and Psychiatry Review, 2, 15-19.

MCCOLL, E., NEWTON, J. \& Hutchinson, A. (1994) An agenda for change in referral-consensus from general practice. British Journal of General Practice. 44, 157162.

Vanstraelen, M. \& Cottrell, D. (1994) Child and adolescent mental health services: purchasers knowledge and plans. British Medical Journal, 309. 259-261.

WHITFELD, M. (1997) Giving feedback to questionnaire responders - an essential task? British Journal of General Practice, 47, 241-243.

WOLPERT, M. \& FREDMAN, G. (1996) Child characteristics influencing referral to mental health services. Child Psychology and Psychiatry Review. 1, 98-103.

*Stephen M. Jones. Senior Registrar in Child and Adolescent Psychiatry and B. R. Bhadrinath. Consultant Child and Adolescent Psychiatrist. Bethel Child and Family Centre, Mary Chapman House, Hotblack Road, Norwich, Norfolk NR2 4HN

*Correspondence 